\title{
A CROSS-SECTIONAL STUDY OF THE UPPER LIMB NON-NEUROGENIC PHYSICAL FINDINGS IN COMPUTER OPERATORS AND THEIR RELATION TO PAIN AND NEUROLOGICAL FINDINGS
}

\author{
KASPER RIIS JEPSEN, GERT FRANK THOMSEN, and JØRGEN RIIS JEPSEN
}

\author{
Hospital South West Jutland, Esbjerg, Denmark \\ Department of Occupational Medicine
}

\begin{abstract}
Objectives: The character of upper limb disorders in computer operators is subject to debate. While nerve involvement is suggested by the presence of pain, paresthesia and subjective weakness, these symptoms are mainly interpreted as related to pathologies outside the nervous system. Findings in a previous study involving computer operators indicated peripheral nerve afflictions with specific locations in symptomatic subjects. Based on the same sample, this study addresses the relation of non-neurogenic findings to pain and neurological findings. Material and Methods: Overall, 96 computer operators scored their perceived pain in the neck, shoulder, elbow, and wrist/hand on a Visual Analogue Scale of 0-9. They underwent 2 sets of blinded physical examinations of selected non-neurogenic and neurological items, respectively. The authors analyzed correlations between the scores of each non-neuropathic finding, and a) mean pain scores for each and all regions, and b) scores for neurological patterns reflecting brachial plexopathy, median neuropathy (the elbow), and posterior interosseous neuropathy, respectively, and their combination. Kendall's rank correlation test was applied for all statistical analyses. Results: A median pain level of 1 or 0.5 was reported by 80 and 57 participants on the mouse-operating or contralateral side, respectively. Non-neurogenic and neurological findings were frequent. The mean overall pain correlated with palpation soreness of the neck insertions, and of the trapezius and supraspinatus muscles. Neck and elbow pain correlated with palpation soreness at the neck insertions and the lateral epicondyles, respectively. Significant correlations on the mouse-operating side were identified between posterior interosseous neuropathy and lateral epicondyle soreness, and between median neuropathy and any neurological pattern, and trapezius and lateral epicondyle soreness. Conclusions: Pain correlated with palpation soreness, which again correlated with the neurological patterns. Palpation soreness may be less significant as a marker of a painful disorder as it correlated no better with regional than with overall pain. The physical examination of computer operators should include a sufficient neurological assessment. Int J Occup Med Environ Health. 2021;34(5):679-91
\end{abstract}

Key words:

pain, comorbidity, physical examination, computer operation, musculoskeletal disorders, neuropathy

Received: September 27, 2020. Accepted: February 12, 2021

Corresponding author: Jørgen Riis Jepsen, Hospital South West Jutland, Department of Occupational Medicine, Finsensgade 35, DK-6700 Esbjerg, Denmark (e-mail: jriisjepsen@me.com). 


\section{INTRODUCTION}

About two-thirds of employees in industrialized countries use a computer on a daily basis. For 1 out of 5, computer work represents at least three-quarters of their total working time. Although a recent meta-analysis has delivered heterogeneous evidence for an increased musculoskeletal risk with screen work [1], there is a general consensus that intensive computer work may cause upper limb pain. Major diagnostic challenges characterize a proportion of work-related upper limb disorders including those connected with the use of computers, even when patients experience severe upper limb pain [2]. However, the relation of upper limb pain in computer operators to a clinically identified disease, or to physical findings that may reflect upper limb disorders, remains controversial.

In the absence of physical signs of a disease, computer-related upper limb pain has been characterized by non-specific diagnostic acronyms, such as cumulative trauma disorder or repetition strain injury. Therefore, there is a need for a common understanding with regard to the type and location of the pathology concerned.

Clinicians do not agree on the selection of physical tests, which are likely to reflect complaints. Matters are further complicated by indications that somatization may act as a possible confounder or effect modifier in studies of occupational risk factors in non-specific arm pain [3].

Physical findings relating to pathologies involving the non-neurogenic tissue as well as the peripheral nerves may be present in computer operators' painful limbs. In the limbs in which physical non-neurogenic findings can be identified while neurological findings are absent, pain is more likely centered where the findings are located. Such findings may reflect soft tissue pathology, which may precede subsequent development of nerve afflictions in the same area. Non-neurogenic findings in combination with neurological findings may occur as a co-morbidity, but may also reflect a neurological complication of the identified non-neurogenic condition or a non-neurogenic complication of a neurological condition. If so, these findings are likely related to pain in the limb, but not necessarily to pain where the physical non-neurogenic findings are located.

Thus, the challenge is whether pain perceived by computer operators reflects peripheral nerve afflictions, other soft tissue conditions or their combination. The authors have approached this challenge by analyzing the presence of non-neurogenic physical abnormalities in the same sample of computer operators in active work for which they previously published the neurological findings [4].

The outcomes of previous studies of computer operators that incorporated an assessment of the function of the upper limb peripheral nerves stand in contrast to those of most other researchers. A careful neurological examination of a series of 21 heavily exposed computer operators with serious upper limb symptoms (pain, paresthesia, and/or subjective weakness) referred for an assessment in a hospital department of occupational medicine permitted the identification of rather clear neurological patterns in all patients, suggesting peripheral nerve afflictions with characteristic locations. These included infraclavicular brachial plexus, median nerve at the elbow level, and posterior interosseous nerve (Table 1). According to general diagnostic criteria, no non-neurological disorder could be diagnosed in this sample of patients [5].

A subsequent study supported the role of peripheral neuropathy. A physical examination of 96 "healthy" computer operators in active work identified minor neurological abnormalities consistent with neuropathy in the same 3 locations, suggesting these locations to be characteristic of computer-related upper limb disorders. Workers in this sample complained of pain, paresthesia, and subjective weakness in 67, 23, and 7 limbs, respectively, but their reported symptoms were modest. The cumulated pain score correlated significantly with the individual and summarized scores for the 3 defined neurological patterns (Table 1). There was a higher summarized pain score in 
Table 1. Neurological patterns defined to reflect brachial plexopathy, median neuropathy at the elbow level, and posterior interosseous neuropathy in computer operators $(\mathrm{N}=96)$ from an engineering company in Denmark

\begin{tabular}{llll}
\hline \multirow{2}{*}{ Neuropathy location } & \multicolumn{3}{c}{ Item contained in the model } \\
\cline { 2 - 4 } $\begin{array}{c}\text { Brachial plexopathy } \\
\text { (the chord level) }\end{array}$ & $\begin{array}{l}\text { deltoid } \\
\text { biceps brachii } \\
\text { radial flexor of the wrist }\end{array}$ & $\begin{array}{l}\text { infraclavicular brachial plexus } \\
\text { (the chord level) }\end{array}$ & median nerve \\
$\begin{array}{c}\text { Median neuropathy } \\
\text { (the elbow level) }\end{array}$ & radial flexor of the wrist & median nerve (the elbow level) & median nerve \\
$\begin{array}{c}\text { Posterior interosseous } \\
\text { neuropathy }\end{array}$ & ulnar extensor of the wrist & $\begin{array}{l}\text { posterior interosseous nerve } \\
\text { (the arcade of Frohse) }\end{array}$ & - \\
\hline
\end{tabular}

the mouse-operating limbs than in the non-mouse-operating limbs [4]. These studies indicated that peripheral nerve afflictions contributed to upper limb symptoms in computer operators.

The neurological patterns were defined in accordance with the topography of the upper limb nerves, and their muscular and sensory innervation. The reproducibility of the applied detailed semi-quantitative upper limb neurological examination was demonstrated in a sample of patients both with and without upper limb complaints, referred to a hospital department of occupational medicine. This examination included an assessment of the function of selected muscles while positioning the limb in order to favor a specific muscle and to reduce the influence of others, of the presence of mechanical allodynia with mild pressure along nerve trunks, and of sensory deviations from the normal status in homonymously innervated territories. The physical findings could be reliably integrated into patterns illustrating peripheral nerve afflictions with specific locations. The presence of these patterns was related to the presence of symptoms [6].

A priori, the authors elaborated the following 3 hypotheses:

- the total pain scores for the mouse-operating and contralateral limbs are related to the scores of a selection of individual non-neurogenic physical items;
- the individual pain scores for 3 anatomical regions (the neck, the shoulder, and the elbow) on the mouse-operating and contralateral sides are related to the scores of the individual non-neurogenic physical items in each respective region;

- the scores for non-neurogenic individual findings that can be demonstrated to relate to regional pain correlate with the scores forming the 3 previously identified neurological patterns [5] and their combination.

\section{MATERIAL AND METHODS}

The study base consisted of 155 computer operators in 2 divisions of a Danish engineering company. All computer operators were exposed to computer work for $>20 \%$ of their total working time or had experienced upper limb symptoms within the last 12 months. A sub-sample of 96 computer operators (66 engineers and 30 technical assistants) completed a questionnaire about upper limb symptoms and accepted participation in the subsequent physical examinations. This sample is identical to the one previously reported [4].

The study group comprised 38 female computer operators whose median age was 30 years (range: 20-60) and 58 males whose median age was also 30 years (range: 20-50). The median body mass index was 24 (range: 19-44) and 25 (range: 21-33) for females and males, 
respectively, and the median professional computer experience was 122 months (range: 13-492) and 101 months (range: 17-307), respectively.

\section{Questionnaire and physical examination}

A web-questionnaire based on the Nordic Questionnaire [7] included questions about demographics and experienced symptoms including pain in the neck, shoulder, elbow and hand/wrist on both sides. Each anatomical region was defined by drawings. While subjective weakness and paresthesia were registered, pain during the last 3 months was the only reported symptom used in this study. The respondents scored their perceived pain for each region on a Visual Analogue Scale of 0-9. They indicated their dexterity and their preferred hand for the pointing device. More specifically, 82 used the pointing device with their right hand, 3 with their left hand, and 11 used both hands. For the latter participants, the right side was assigned as the mouse-operating limb.

The participants were subjected to 2 sets of physical examinations by 2 experienced examiners. Both were blinded to any patient-related information including the questionnaire data and each other's findings. The 2 examinations took place in rooms separated from each other. No communication occurred during the physical examinations except instructions from the examiner and reactions from the subject to the applied tests. All examinations were assessed quantitatively.

One examiner studied selected neurological parameters in an examination protocol, which had previously been presented, described in detail and validated [6]. The neurological parameters included those required for neurological patterns defined to illustrate 3 locations of neuropathy (Table 1). The neurological parameters were scored as follows:

Manual testing of individual muscles was quantified [8]:

- contraction against powerful resistance/normal function -0 (the score in [8]: 5),
- contraction against gravity and strong resistance - 1 (the score in [8]: 4+),

- contraction against gravity and moderate resistance -2 (the score in [8]: 4),

- contraction against gravity and slight resistance - 3 (the score in [8]: 4-).

Mechanosensitivity with slight pressure along nerve trunks:

- no soreness -0 ,

- mild mechanical allodynia -1,

- moderate mechanical allodynia -2,

- severe mechanical allodynia - 3 .

Sensibility examined by needle prick (algesia):

- normal sensibility - 0 ,

- reduced/changed sensibility - 1,

- severely reduced/changed sensibility -2 .

The other examiner studied non-neurogenic physical parameters in the neck, shoulder, and elbow using a standard approach as reported elsewhere [9]. This examination addressed the range of motion, palpatory soreness and selected physical tests. The assessed active ranges of motion were dichotomized into normal -0 or reduced -1 . Palpatory soreness was subdivided into none -0 , slight -1 , moderate -2 , or pronounced with jump sign -3 . Each test (the foraminal compression test, the impingement test, and Yergason's test) was scored as normal -0 or positive -1 (Table 2 ).

\section{Statistics}

For each limb, a new variable was created from the mean of the scores for perceived pain during the last 3 months on the mouse-operating and non-mouse-operating sides in the neck, shoulders, elbows, and hand/wrists. The resulting mean scores for pain in each limb would be in the range of 0 (no pain at all) to 9 (severe pain in all 4 regions). The score for neck pain was included in the resulting mean score on both sides.

For each limb, Kendall's rank correlation test was applied to analyze the relations between the mean pain score for 
Table 2. Relation of pain scores for all regions to the scores from the physical examination of non-neurogenic qualities in computer operators from an engineering company in Denmark

\begin{tabular}{|c|c|c|c|c|c|c|c|c|}
\hline \multirow{3}{*}{ Examined quality } & \multicolumn{8}{|c|}{$\begin{array}{l}\text { Participants } \\
(\mathrm{N}=96)\end{array}$} \\
\hline & \multicolumn{4}{|c|}{ mouse-operating limb } & \multicolumn{4}{|c|}{ non-mouse-operating limb } \\
\hline & $\begin{array}{l}\operatorname{limbs} \\
{[\mathrm{n}]}\end{array}$ & $\begin{array}{l}\text { pain } \\
(\mathrm{Me})\end{array}$ & $\begin{array}{l}\text { Kendall's } \\
\text { tau-b }\end{array}$ & $\mathrm{p}$ & $\begin{array}{c}\operatorname{limbs} \\
{[\mathrm{n}]}\end{array}$ & $\begin{array}{l}\text { pain } \\
(\mathrm{Me})\end{array}$ & $\begin{array}{l}\text { Kendall's } \\
\text { tau-b }\end{array}$ & $\mathrm{p}$ \\
\hline \multicolumn{9}{|l|}{ Neck } \\
\hline $\begin{array}{l}\text { rotation (movement towards the side): normal }-0 \text {, } \\
\text { reduced }(<60)-1\end{array}$ & 8 & 2.5 & 0.05 & 0.60 & 3 & 3.5 & 0.13 & 0.16 \\
\hline flexion: normal -0 , reduced $(<40)-1$ & 3 & 1.5 & 0.07 & 0.46 & 3 & 1.5 & 0.90 & 0.32 \\
\hline extension: normal -0 , reduced $(<45)-1$ & 3 & 3.5 & 0.05 & 0.61 & 3 & 3.5 & 0.21 & 0.24 \\
\hline $\begin{array}{l}\text { lateral bending (movement towards the side): normal }-0 \text {, } \\
\text { reduced }(<55)-1\end{array}$ & 64 & 1 & 0.05 & 0.56 & 64 & 0.5 & 0.16 & 0.08 \\
\hline $\begin{array}{l}\text { foraminal compression test (movement towards the side): } \\
\text { normal - } 0 \text {, positive (radiating pain) }-1\end{array}$ & 0 & & & & 0 & & & \\
\hline $\begin{array}{l}\text { neck insertions (palpation soreness): none }-0 \text {, slight }-1 \text {, } \\
\text { moderate }-2 \text {, pronounced }-3\end{array}$ & 71 & 1.5 & 0.32 & 0.0001 & 57 & 0.5 & 0.25 & 0.0035 \\
\hline \multicolumn{9}{|l|}{ Shoulder } \\
\hline collision pain/impingement: no pain -0 , collision pain -1 & 0 & & & & 0 & & & \\
\hline pain arch $80-120$ degrees: no pain -0 , shoulder pain -1 & 0 & & & & 2 & 2.5 & & \\
\hline Yergason's test: no pain -0 , shoulder pain -1 & 1 & 3 & & & 2 & 2 & & \\
\hline abduction: $>150-0,0-150-1$ & 0 & & & & 0 & & & \\
\hline flexion: >180 - 0, 0-180-1 & 32 & 1 & -0.02 & 0.85 & 32 & 0.5 & -0.02 & 0.82 \\
\hline extension: $>45-0,0-45-1$ & 5 & 1.5 & 0.14 & 0.12 & 6 & 1 & 0.16 & 0.07 \\
\hline internal rotation: $>90-0,0-90-1$ & 0 & & & & 0 & & & \\
\hline external rotation: $>45-0,0-45-1$ & 7 & 1 & -0.01 & 0.88 & 3 & 0 & -0.11 & 0.23 \\
\hline $\begin{array}{l}\text { trapezius (palpation soreness): none }-0 \text {, slight }-1 \text {, } \\
\text { moderate }-2 \text {, pronounced }-3\end{array}$ & 60 & 1.5 & 0.24 & 0.003 & 55 & 1 & 0.26 & 0.003 \\
\hline $\begin{array}{l}\text { infraspinatus (palpation soreness): none }-0 \text {, slight }-1 \text {, } \\
\text { moderate }-2 \text {, pronounced }-3\end{array}$ & 9 & 1.5 & 0.10 & 0.28 & 8 & 1 & 0.12 & 0.20 \\
\hline $\begin{array}{l}\text { supraspinatus (palpation soreness): none }-0 \text {, slight }-1 \text {, } \\
\text { moderate }-2 \text {, pronounced }-3\end{array}$ & 24 & 1.5 & 0.20 & 0.02 & 12 & 1 & 0.24 & 0.01 \\
\hline \multicolumn{9}{|l|}{ Elbow } \\
\hline $\begin{array}{l}\text { lateral epicondyle (palpation soreness): none }-0 \text {, slight }-1 \text {, } \\
\text { moderate }-2 \text {, pronounced }-3\end{array}$ & 36 & 1.5 & 0.09 & 0.30 & 29 & 1 & 0.24 & 0.008 \\
\hline passive wrist flexion: normal -0 , elbow pain -1 & 12 & 1 & & & 3 & 2.5 & & \\
\hline isometric wrist extension: normal - 0 , elbow pain -1 & 0 & & & & 0 & & & \\
\hline $\begin{array}{l}\text { medial epicondyle (palpation soreness): none }-0 \text {, slight }-1 \text {, } \\
\text { moderate }-2 \text {, pronounced }-3\end{array}$ & 15 & 2 & 0.19 & 0.03 & 14 & 1 & 0.14 & 0.13 \\
\hline
\end{tabular}

Bolded are significant values. 


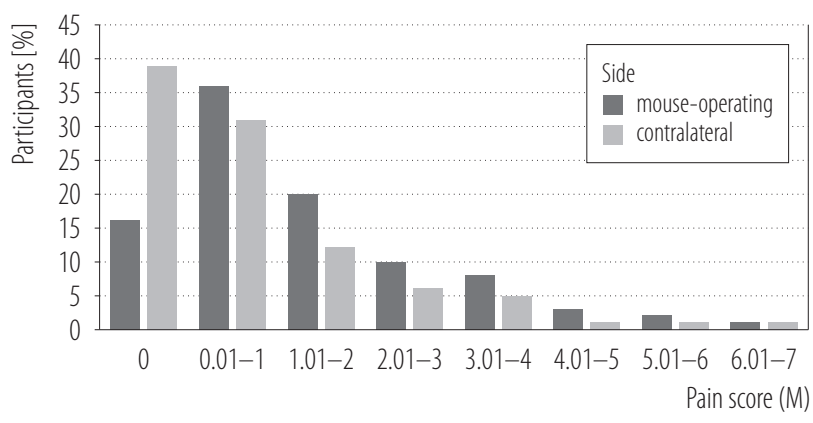

Figure 1. Distribution of perceived pain (pain scoring options in the interval of 0-9) in the mouse-operating and non-mouse-operating limb in computer operators $(\mathrm{N}=96)$ from an engineering company in Denmark

all 4 regions and the scores for each non-neurological item, and the relation between scores for pain in each of the 4 regions and the score for physical non-neurogenic findings in the same region.

New variables reflecting infraclavicular brachial plexopathy (the chord level), median neuropathy (the elbow) and posterior interosseous neuropathy were defined according to Table 1 . The mean scores for each component that contributes to the patterns were calculated for each limb, i.e., the scores for contraction reflecting each muscle, for sensibility (algesia) in each territory, and for mechanosensitivity in each location, respectively, as relevant for each individual pattern. The relation between the scores for the non-neurogenic physical findings and the mean scores illustrating each of the 3 patterns and their combination was studied by Kendall's rank correlation test.

The significance level for all statistical analyses was set at 0.05 . By defining the level of significance according to the number of items examined, adjustments were made for a potential mass significance (the Bonferroni adjustment) [10]. Data were processed by Stata v. 14.

\section{Ethics}

The study complied with the Helsinki Declaration. It was approved by the local Ethics Committee (2487A-03) and signed informed consent was obtained from all participants.

\section{RESULTS}

\section{Prevalence of perceived pain}

Perceived pain during the last 3 months in the mouse-operating and contralateral limbs was reported by 80 and 57 study subjects, respectively. With scoring options in the interval of $0-9$, pain was limited in this sample of healthy computer operators (a median score of 1 [range: 0-7] and 0.5 [range: 0-7], respectively, on the 2 sides). The perceived level of pain was clearly higher in the mouse-operating limb than contralaterally (Figure 1). Overall, 53 subjects experienced neck pain, 44 and 22 shoulder pain, 15 and 7 elbow pain, and 43 and 10 subjects hand/wrist pain in the mouseoperating or contralateral limb, respectively.

\section{Prevalence of physical findings}

Non-neurogenic physical findings assessed as abnormal were prevalent, in particular on the mouse-operating side, but of a minor intensity (Table 2). Generally, the median pain intensity was higher when abnormal findings were identified. In this sample of computer operators, some physical abnormalities were frequent while other items were rarely (pain arch, Yergason's test, passive wrist flexion and isometric wrist extension), or never abnormal (the foraminal compression test, collision pain/impingement, and a reduced range of abduction, internal rotation) (Table 2). The latter outcomes were excluded from statistical calculations.

\section{Total mean pain score}

\section{vs. non-neurogenic physical findings}

With the level of significance set at 0.05 , only palpation soreness correlated significantly with the mean pain scores for all 4 regions taken together, in particular on the mouseoperating side: the neck insertions, and the trapezius and supraspinatus muscles on both sides, the medial epicondyle on the mouse-operating side, and the lateral epicondyle on the non-mouse-operating side (Table 2). Following the Bonferroni adjustment, palpation soreness on the medial epicondyle became non-significant. 
Table 3. Relation between pain scores for the neck, shoulder and elbow to scores for palpation soreness in 4 locations in computer operators from an engineering company in Denmark

\begin{tabular}{|c|c|c|c|c|}
\hline \multirow{3}{*}{ Pain location/Palpation soreness } & \multicolumn{4}{|c|}{$\begin{array}{l}\text { Participants } \\
\quad(\mathrm{N}=96)\end{array}$} \\
\hline & \multicolumn{2}{|c|}{ mouse-operating limb } & \multicolumn{2}{|c|}{ non-mouse-operating limb } \\
\hline & Kendall's tau-b & $\mathrm{p}$ & Kendall's tau-b & $\mathrm{p}$ \\
\hline \multicolumn{5}{|l|}{ Neck } \\
\hline neck insertions & 0.25 & 0.0029 & 0.24 & 0.0053 \\
\hline \multicolumn{5}{|l|}{ Shoulder } \\
\hline trapezius muscle & 0.07 & 0.44 & 0.11 & 0.22 \\
\hline supraspinatus muscle & 0.08 & 0.37 & 0.18 & 0.64 \\
\hline \multicolumn{5}{|l|}{ Elbow } \\
\hline lateral epicondyle & 0.19 & 0.03 & 0.29 & 0.0017 \\
\hline
\end{tabular}

Bolded are significant values.

\section{Mean pain score in each region}

\section{vs. non-neurogenic physical findings}

Neck pain was significantly related to the scores for palpation soreness at the neck insertions, and elbow pain to palpation soreness of the lateral epicondyles. Following the Bonferroni adjustment, the latter correlation remained significant on the non-mouse-operating side. Palpation soreness of the trapezius and infraspinatus muscles was unrelated to shoulder pain (Table 3).

On the mouse-operating side, 10 subjects experienced soreness at the lateral epicondyle in combination with pain provocation on passive wrist flexion, suggesting the diagnosis of lateral epicondylitis; 2 of them additionally had this combination contralaterally. However, the criteria defined to reflect posterior interosseous neuropathy (radial tunnel syndrome) (Table 1) were fulfilled for 9 of these subjects in the dominant limb and for 2 subjects on the contralateral side.

\section{Correlation between scores}

for non-neurogenic physical findings

and scores for neurological patterns

The neurological findings have previously been reported in detail [4]. Weakness, hypalgesia and mechanical nerve trunk allodynia were identified in 56 and 25, 40 and 9, and 60 and 34 mouse-operating or non-mouse-operating limbs, respectively. According to the defined criteria (Table 1), brachial plexopathy, posterior interosseous neuropathy, and median neuropathy at the elbow level were found in 9 and 2, 14 and 8, and 13 and 5 mouse-operating or nonmouse-operating limbs, respectively.

The scores for infraclavicular brachial plexopathy correlated significantly with palpation soreness at the neck insertions and the trapezius muscle. Both correlations lost significance following the Bonferroni adjustment.

Correlations were also found between posterior interosseous neuropathy and palpation soreness of the trapezius muscle and the lateral epicondyle on the mouse-operating side, as well as soreness of the neck insertions on the nonmouse-operating side. Palpation soreness of the lateral epicondyle remained significant following the Bonferroni adjustment.

Median neuropathy at the elbow level was significantly related to soreness of the trapezius muscle bilaterally, soreness of the neck insertions and the supraspinatus muscle on the non-mouse-operating side, and soreness of the lateral epicondyle on the mouse-operating side. 
Table 4. Relation of non-neurogenic findings to scores defining the 3 neurological patterns in computer operators from an engineering company in Denmark

\begin{tabular}{|c|c|c|c|c|}
\hline \multirow{3}{*}{ Neurological patterns/palpation soreness } & \multicolumn{4}{|c|}{$\begin{array}{l}\text { Participants } \\
\quad(\mathrm{N}=96)\end{array}$} \\
\hline & \multicolumn{2}{|c|}{ mouse-operating limb } & \multicolumn{2}{|c|}{ non-mouse-operating limb } \\
\hline & Kendall's tau-b & $\mathrm{p}$ & Kendall's tau-b & $\mathrm{p}$ \\
\hline \multicolumn{5}{|l|}{ Brachial plexus, the chord level } \\
\hline neck insertions & 0.19 & 0.03 & 0.21 & 0.03 \\
\hline trapezius muscle & 0.25 & 0.004 & 0.18 & 0.05 \\
\hline supraspinatus muscle & 0.03 & 0.71 & -0.02 & 0.84 \\
\hline lateral epicondyle & 0.17 & 0.06 & 0.10 & 0.31 \\
\hline \multicolumn{5}{|l|}{ Posterior interosseous nerve } \\
\hline neck insertions & 0.09 & 0.32 & 0.21 & 0.03 \\
\hline trapezius muscle & 0.19 & 0.03 & 0.11 & 0.24 \\
\hline supraspinatus muscle & 0.12 & 0.21 & 0.14 & 0.17 \\
\hline lateral epicondyle & 0.30 & 0.0009 & 0.08 & 0.40 \\
\hline \multicolumn{5}{|l|}{ Median nerve, the elbow level } \\
\hline neck insertions & 0.15 & 0.09 & 0.32 & 0.0006 \\
\hline trapezius muscle & 0.24 & 0.006 & 0.20 & 0.03 \\
\hline supraspinatus muscle & 0.12 & 0.19 & 0.21 & 0.03 \\
\hline lateral epicondyle & 0.23 & 0.01 & 0.15 & 0.12 \\
\hline \multicolumn{5}{|l|}{ Any neurological pattern } \\
\hline neck insertions & 0.15 & 0.08 & 0.21 & 0.02 \\
\hline trapezius muscle & 0.23 & 0.006 & 0.15 & 0.08 \\
\hline supraspinatus muscle & 0.11 & 0.22 & 0.17 & 0.07 \\
\hline lateral epicondyle & 0.23 & 0.007 & 0.10 & 0.29 \\
\hline
\end{tabular}

Bolded are significant values.

Correlations for the trapezius muscle and the lateral epicondyle remained significant following the Bonferroni adjustment.

Correlations with any neurological pattern were present for soreness of the neck insertions on the non-mouseoperating side, and soreness of the trapezius muscle and the lateral epicondyle on the mouse-operating side. Following the Bonferroni adjustment, significance remained for the trapezius muscle and the lateral epicondyle on the mouse-operating side (Table 4).

\section{DISCUSSION}

According to the applied criteria, a considerable proportion of physical outcomes in the studied sample of computer operators were abnormal although the actively working computer operators included in this sample were of relatively young age and characterized by less perceived upper limb pain than reported elsewhere [2,11-13]. In addition to a high frequency of neurological findings [4], palpation soreness was common and related to perceived overall pain, in particular for the neck insertions, the trapezius 
and supraspinatus muscles, and the epicondyles (Table 2). A reduced range of shoulder and neck motion was also seen (Table 2), but it was unrelated to pain. Regional neck and elbow pain correlated with palpation soreness of the neck insertions and the lateral epicondyle, respectively. Following the Bonferroni adjustment, significant correlations remained for palpation soreness of the lateral epicondyle, and posterior interosseous neuropathy and any neurological patterns combined, and for palpation soreness of the trapezius and the lateral epicondyle for median neuropathy and for any neurological patterns (Table 4). Calculating the mean of the perceived pain for several regions assumes that experiencing a minor pain in several regions may equal severe pain in a single region, which may not be the case. A similar concern may arise from adding the scores from the neurological assessment which assumes, e.g., that a score of 3 for muscular strength equals the scores of 1 for muscular strength, 1 for sensibility, and 1 for nerve trunk allodynia. However, the mean overall pain does indicate the level of pain, and the added scores for neurological findings are practical measures for this study, but evidently less so for clinical assessments. As the relations between palpation soreness and the overall pain had the same direction, significant relations can hardly be due to mass significance [10].

Conventionally, regional physical findings tend to be linked to pain in the same region. It is, therefore, relevant to compare physical findings to the reported perceptions of regional pain, i.e., pain in the area where palpation soreness was found. The observation that palpation soreness correlated no better with regional pain than with overall pain (Tables 2 and 3) challenges the significance of palpation soreness in the studied locations as a marker of a painful disorder. It is also noteworthy that the identification of lateral epicondylitis as defined by palpation soreness at the lateral epicondyle, in combination with painful passive wrist flexion, was almost fully in accordance with the presence of posterior interosseous neuropathy as defined in Table 1.
It is well known that the pathology in the upper limb conditions may be situated distantly to the dominant location of pain, in particular in neuropathic conditions. This is supported by a previous study which confirmed the assumption that peripheral neuropathy in the 3 defined locations (infraclavicular brachial plexus, posterior interosseous nerve, and median nerve at the elbow level) was involved in computer-related upper limb disorders and connected with the level of perceived pain with any location [4].

The identification of minor physical abnormalities in many participants with minimal or no upper limb pain may question the validity of the physical examination. Positive findings in the absence of pain may be due to a highly sensitive physical examination the outcome of which relates not only to a symptomatic disorder but potentially also to a pre-clinical minor dysfunction. The defined neurological patterns have been demonstrated to correlate with perceived pain [6]. While palpation soreness of the neck insertions is related to neck pain and that of the lateral epicondyle to elbow pain (Table 3), palpation soreness at these locations was also shown to correlate with neurological patterns (Table 4). These non-neurological findings may precede neurological findings, represent co-morbidity or develop consequent to the neurological conditions.

All correlations between non-neurogenic findings and the 3 neurological patterns were low to modest, but all except supraspinatus muscle soreness and brachial plexopathy on the non-mouse-operating side were positive (Table 4). This observation indicates that both sets of findings reflect a common pathology. The correlations in Table 4 suggest that brachial plexopathy is linked to palpation soreness of the neck insertions and the trapezius muscle. This can hardly be explained by a muscle-related condition causing direct external compromise of the brachial plexus due to the distance between these 2, but rather by shared risk factors. The significant overlap between lateral epicondylitis and posterior interosseous neuropathy is less surprising and suggests the relationship between 
these 2 conditions. Palpation soreness of the neck insertions (the non-mouse-operating side) and the trapezius muscle (the mouse-operating side) with posterior interosseous neuropathy and the neck insertions, the trapezius (bilaterally) and supraspinatus muscle (the non-mouseoperating side) and lateral epicondylitis (the mouse-operating side) with median neuropathy is more spurious, but may also be related to shared risk factors for a muscular as well as neural compromise.

Muscle soreness is likely to accompany an adverse muscular balance with anterior shoulder muscles (e.g., the pectorals) shortened and other muscles (the upper trapezius) overused. Such an imbalance characterizes many workrelated conditions including those affecting office workers [14], and tend to put the brachial plexus as well as the peripheral upper limb nerves at risk [15].

There was a higher prevalence of symptoms and abnormal findings on the mouse-operating side than contralaterally. The observation of similar non-neurogenic and neurological abnormalities at the 2 sides is also noteworthy. Since the early descriptions on scriveners by Ramazzinus [16], the tendency to the contralateral spread of work-related upper limb pain has been acknowledged by many clinicians. This phenomenon may be due to factors such as substitution by work being taken over by the intact limb, sensitization of the upper limb nerves secondary to neurogenic inflammation, and central processing of sensory inputs. With computer work, a similar exposure from keyboarding with both hands, or using a mouse in one and a digitizer in the other hand, may also contribute to the findings.

All physical assessments and the interpretation of findings were performed while blinded to any information about the studied computer operators. In addition, the 2 examiners of non-neurogenic and neurological physical items, respectively, were blinded to the findings of each other. The blinding aimed to reduce subjectivity of the examiners. Still, it cannot be excluded that one finding, whether of a non-neurogenic or neuropathic character, can bias the subsequent findings by the same examiner. Such a bias is likely to be non-differential and to have limited influence on the results.

Even if the perception of pain in this study of a mostly healthy group of computer operators may be influenced by psychological factors, the physical findings are less likely affected for a number of reasons: some non-neuropathic as well as neurological abnormalities were also frequent in subjects without symptoms; e.g., reduced muscle function was identified in 56 mouse-operating limbs out of which 13 were completely without pain and only 7 had subjective weakness [4]. Reduced muscle strength in the absence of subjective weakness is commonly found in upper limb patients including computer operators [5].

The authors noticed on many occasions the surprise of patients with no complaints of weakness when, during testing, they clearly perceived reduced strength of which they had been previously unaware. When asked to simultaneously do their best on both sides, the examinees would not be likely to deliberately exert less force than to their capability, and with an actual identical sensibility, they would hardly report a perceived difference in 2 compared territories. While the presence of a more severe allodynia is readily visible from the subject's reaction, the examinees could hardly be expected to exaggerate the subjective level of minor soreness as this study deals with active "healthy" subjects in an attractive enterprise. Exaggerating or malingering would not provide them any benefit. The selectivity of non-neurogenic as well as neurological findings, e.g., a weakness tending to affect certain muscles with others remaining normal and the rarity of nerveafflictions elsewhere than in the 3 locations mentioned argues against a major role of a psychological reaction. Most importantly, for the examinees constructing the neurological patterns requires a less plausible familiarity with anatomical features such as the innervation patterns and topography of nerves. 


\section{Previous studies}

Previous studies of upper limb disorders in computer operators have reached conflicting results. The physical examination in a cross-sectional study of almost 7000 computer operators working intensively with pointing devices identified minimal clinical disease, similar to what would be expected in the general population. During the preceding week, $10.7 \%$ had moderate to severe pain in the neck, and $7.7 \%$ in the right shoulder. However, apart from a possible relation to tension neck syndrome, the physical examination of symptomatic workers failed to identify clinical disorders responsible for their complaints $[2,17]$. Numbness and/or tingling was reported by $10.9 \%$, and it was located in the median nerve territory in $4.8 \%$ of these cases. Nocturnal symptoms in $1.4 \%$ of cases could potentially be attributed to carpal tunnel syndrome but were unexplained in the remaining subjects [18]. Defined by the localized palpation tenderness with withdrawal and pain with provocative maneuvers, nerve entrapments were diagnosed in 12 subjects. At follow-up after 1 year, no new cases were identified [19].

This study stands in contrast to a study of 533 computer workers among whom upper limb disorders were diagnosed in $22 \%$, and were dominated by tendon- and muscle-related conditions ( $15 \%$ and $8 \%$, respectively), and probable nerve entrapment in 4\% [12]. Among 632 newly hired computer operators involved in another study, a 1-year incidence of $58 \%$ of neck and shoulder, and $39 \%$ of hand/arm symptoms was found. Two diagnoses covered almost all symptomatic cases. Somatic shoulder/neck syndrome was characterized by non-specific signs and an absence of well-defined pathology, and de Quervain's syndrome by a positive Finkelstein test, which, however, is neither pathognomonic nor specific for this condition [13]. Diagnostics, however, depends on the selection and validity of the applied clinical tests and diagnostic criteria. These quoted studies had no detailed neurological upper limb assessment included in their examination protocols.
The identification of palpation soreness of the neck insertions and shoulder muscles, which was obtained in the current study, supports the findings of tension neck/somatic shoulder/neck syndrome, but the authors found no signs that would be consistent with de Quervain's syndrome. Nor did they find any clear signs suggesting a tendon-related disorder (lateral epicondylitis) which, however, was generally combined with posterior interosseous neuropathy.

Similar nerve conduction velocity and vibrotactile perception were found in healthy computer users and non-exposed controls [20]. Other studies identified sub-clinical median nerve impairment [21] and elevated thresholds for the perception of vibration $[22,23]$ persisting along with symptoms [24]. One study demonstrated a $15 \%$ increased vibration threshold in computer operators with paresthesia in contrast to those without paresthesia [25]. Among 485 upper limb patients ( $70 \%$ computer operators), a neurogenic thoracic outlet syndrome was suggested in $70 \%$ by tests stressing the brachial plexus and by the demonstration of localized mechanical nerve trunk allodynia [26].

Combined with clinical experiences, findings such as abnormal reactions to upper limb tension tests, reduced nerve mobility and mechanical allodynia with minor pressure over nerve trunks may represent reactions to lesions or loading of peripheral nerves [15,27-30]. These observations suggest the involvement of multiple nerve entrapment in the so-called non-specific upper limb conditions such as those occurring in office employees [14].

\section{CONCLUSIONS}

This study of computer operators has identified some non-neurogenic physical abnormalities as well as individual neurological findings and patterns. While the latter seems to reflect afflictions of the upper limb peripheral nerves with specific locations (brachial plexus at the chord level, the posterior interosseous and median nerves at the elbow level) [4,5], the demonstrated relation to upper limb pain of the findings such as palpation soreness has 
a less clear role and may represent comorbidity, predisposing factors or secondary features reflecting various stages of the disorder.

This study highlights the importance of neurological physical findings for the diagnosis of work-related upper limb disorders such as those occurring in computer operators. The practical implication of this study would, therefore, be the inclusion of selected neurological items in the physical examination. Even though a comprehensive physical examination of the function of the upper limb peripheral nerves is easy to learn and quite rapid to execute, it may appear overwhelming for some clinicians.

The authors, therefore, suggest a simple initial approach consisting of manual testing of the strength in the following 3 antagonist muscle pairs: pectorals - posterior deltoid, biceps - triceps, and radial flexor of the wrist - short extensor of the wrist. These muscles are easy to both remember and test. With any weakness in these muscles, the examiner should proceed with an examination of further neurological items. This methodology of manual muscle testing has been described in detail in previous publications $[4,6]$. The sensitivity of the neurological examination seems to be high, since it is capable of identifying a high number of neurological abnormalities even in non-symptomatic subjects. This observation suggests that neurological parameters may be useful for screening the populations at risk.

\section{REFERENCES}

1. Coenen P,van der Molen HF, Burdorf A, Huysmans MA, Straker L, Frings-Dresen MH, et al. Associations of screen work with neck and upper extremity symptoms: a systematic review with meta-analysis. Occup Environ Med. 2019;76(7):502-9, https://doi.org/10.1136/oemed-2018-105553.

2. Lassen CF, Mikkelsen S, Kryger AI, Brandt LP, Overgaard E, Thomsen JF, et al. Elbow and wrist/hand symptoms among 6,943 computer operators: a 1-year follow-up study (the NUDATA study). Am J Ind Med. 2004;46(5):521-33, https://doi. org/10.1002/ajim.20081.
3. Palmer KT, Calnan M, Wainwright D, Poole J, O'Neill C, Winterbottom A, et al. Disabling musculoskeletal pain and its relation to somatization: a community-based postal survey. Occup Med (Lond). 2005;55(8):612-7, https://doi.org/ 10.1093/occmed/kqi142.

4. Jepsen JR, Thomsen G. A cross-sectional study of the relation between symptoms and physical findings in computer operators. BMC Neurology. 2006;6:40, https://doi.org/10. 1186/1471-2377-6-40.

5. Jepsen JR. Upper limb neuropathy in computer operators? A clinical case study of 21 patients. BMC Musculoskel Disord. 2004;5:26, https://doi.org/10.1186/1471-2474-5-26.

6. Jepsen JR. Studies of upper limb pain in occupational medicine, in general practice, and among computer operators [dissertation]. Dan Med J. 2018;65(4):1-41.

7. Kuorinka I, Jonsson B, Kilbom A, Vinterberg H, Biering-Sorensen F, Andersson G, et al. Standardised Nordic questionnaires for the analysis of musculoskeletal symptoms. Appl Ergon. 1987;18(3):233-7.

8. The Editorial Commitee for the Guarantors of Brain. Aids to the examination of the peripheral nervous system. London: Ballière Tindall; 1986.

9. Laursen LH, Sjogaard G, Hagert CG, Jepsen JR. Diagnostic distribution of non-traumatic upper limb disorders: vibrotactile sense in the evaluation of structured examination for optimal diagnostic criteria. Med Lav. 2007;98(2):127-44.

10. Bland JM, Altman DG. Multiple significance tests: the Bonferroni method. BMJ. 1995;310(6973):170, https://doi.org/ 10.1136/bmj.310.6973.170.

11. Lassen CF, Mikkelsen S, Kryger AI, Brandt LPA, Overgaard E, Thomsen JF, et al. Elbow and wrist/hand symptoms among 6,943 computer operators: A 1-year follow-up study (The NUDATA study). Am J Ind Med. 2004;46(5):521-33, https://doi.org/10.1002/ajim.20081.

12. Hales TR, Sauter SL, Peterson MR, Fine LJ, Putz-Anderson V, Schleifer LR, et al. Musculoskeletal disorders among visual display terminal users in a telecommunications company. Ergonomics. 1994;37(10):1603-21. 
13. Gerr F, Marcus M, Ensor C, Kleinbaum D, Cohen S, Edwards A, et al. A prospective study of computer users: I. Study design and incidence of musculoskeletal symptoms and disorders. Am J Ind Med. 2002;41(4):221-35.

14. Novak CB, Mackinnon SE. Multiple nerve entrapment syndromes in office workers. Occup Med. 1999;14(1):39-59.

15. Novak CB, Mackinnon SE. Multilevel nerve compression and muscle imbalance in work-related neuromuscular disorders. Am J Ind Med. 2002;41(5):343-52, https://doi.org/10. 1002/ajim.10063.

16. Ramazzinus B. De Morbis Artificum Diatriba. Modena: Antonii Capponi; 1700.

17. Brandt LP, Andersen JH, Lassen CF, Kryger A, Overgaard E, Vilstrup I, et al. Neck and shoulder symptoms and disorders among Danish computer workers. Scand J Work Environ Health. 2004;30(5):399-409.

18. Andersen JH, Thomsen JF, Overgaard E, Lassen CF, Brandt LP, Vilstrup I, et al. Computer use and carpal tunnel syndrome: a 1-year follow-up study. JAMA. 2003;289(22):2963-9.

19. Kryger AI, Andersen JH, Lassen CF, Brandt LP, Vilstrup I, Overgaard E, et al. Does computer use pose an occupational hazard for forearm pain; from the NUDATA study. Occup Environ Med. 2003;60(11):e14.

20. Sanden H, Edblom M, Ekman A, Tenenbaum A, Wallin BG, Hagberg M. Normal nerve conduction velocity and vibrotactile perception thresholds in computer users. Int Arch Occup Environ Health. 2005;78(3):239-42, https://doi.org/10.1007/ s00420-005-0606-X.

21. Murata K, Araki S, Okajima F, Saito Y. Subclinical impairment in the median nerve across the carpal tunnel among female VDT operators. Int Arch Occup Environ Health. 1996;68(2):75-9.

22. Jensen BR, Pilegaard M, Momsen A. Vibrotactile sense and mechanical functional state of the arm and hand among computer users compared with a control group. Int Arch Occup Environ Health. 2002;75(5):332-40, https://doi.org/ 10.1007/s00420-001-0301-5.

23. Greening J, Lynn B. Vibration sense in the upper limb in patients with repetitive strain injury and a group of at-risk office workers. Int Arch Occup Environ Health. 1998;71(1): 29-34.

24. Pilegaard M, Jensen BR. An 18-month follow-up study on vibrotactile sense, muscle strength and symptoms in computer users with and without symptoms. Int Arch Occup Environ Health. 2005;78(6):486-92, https://doi.org/10.1007/ s00420-005-0626-6.

25. Overgaard E, Brandt LP, Ellemann K, Mikkelsen S, Andersen JH. Tingling/numbness in the hands of computer users: neurophysiological findings from the NUDATA study. Int Arch Occup Environ Health. 2004;77(7):521-5, https://doi. org/10.1007/s00420-004-0545-y.

26. Pascarelli EF, Hsu YP. Understanding work-related upper extremity disorders: clinical findings in 485 computer users, musicians, and others. J Occup Rehabil. 2001;11(1):1-21.

27. Mitchell S, Cooper C, Martyn C, Coggon D. Sensory neural processing in work-related upper limb disorders. Occup Med (Lond). 2000;50(1):30-2, https://doi.org/10.1093/ occmed/50.1.30.

28. Elvey RL, Quintner JL, Thomas AN. A clinical study of RSI. Aust Fam Physician. 1986;15(10):1314-5.

29. Armstrong TJ, Buckle P, Fine LJ, Hagberg M, Jonsson B, Kilbom A, et al. A conceptual model for work-related neck and upper-limb musculoskeletal disorders. Scand J Work Environ Health. 1993;19(2):73-84.

30. Rempel D, Dahlin L, Lundborg G. Pathophysiology of nerve compression syndromes: response of peripheral nerves to loading. J Bone Joint Surg Am. 1999;81(11):1600-10, https:// doi.org/10.2106/00004623-199911000-00013.

This work is available in Open Access model and licensed under a Creative Commons Attribution-NonCommercial 3.0 Poland License - http://creativecommons.org/ licenses/by-nc/3.0/pl/deed.en. 